Published in final edited form as:

Curr Probl Cancer. 2016 ; 40(2-4): 141-150. doi:10.1016/j.currproblcancer.2016.09.004.

\title{
Targeting the androgen receptor in triple negative breast cancer
}

\author{
Ayca Gucalp ${ }^{1,2}$ and Tiffany A Traina ${ }^{1,2}$ \\ ${ }^{1}$ Memorial Sloan Kettering Cancer Center, New York, NY \\ ${ }^{2}$ Weill Cornell Medical College, New York, NY
}

\begin{abstract}
Keywords
Triple negative breast cancer; androgen receptor; bicalutamide; enzalutamide; abiraterone acetate; seviteronel
\end{abstract}

\section{Introduction}

Triple negative breast cancer (TNBC), a relatively new term first published in 2005[1], is used to describe a subset of breast cancers characterized by the absence of expression of the estrogen receptor (ER), progesterone receptor (PgR), and HER2 protein. TNBC represents approximately 15-20\% of all newly diagnosed breast cancers but accounts for a disproportionate number of breast cancer-related deaths each year making up 5\% of all cancer deaths annually.[2,3] Due to the lack of receptors, patients with TNBC do not benefit from generally well-tolerated and effective therapies targeting the ER and HER2 and often experience a more aggressive clinical course with increased risk of disease progression and poorer overall survival.[4] A subset of TNBC tumors express the AR and may benefit from treatments that inhibit the AR-signaling pathway. This review summarizes the underlying biology of AR signaling in breast cancer development and the available clinical trial data for the use of antiandrogen therapy in the treatment of AR+ TNBC.

\section{Structure and function of the androgen}

$\mathrm{AR}$ is a member of the steroid-hormone family of receptors which also includes the estrogen, progesterone, glucocorticoid, and mineralocorticoid receptors. Located on chromosome Xq11-12, physiologically AR-regulated signaling is responsible for male sexual differentiation and reproductive development. In adult males, androgens maintain libido, spermatogenesis, muscle mass and strength, bone mineral density, and erythropoiesis. The gene for AR encodes a 110kDa polypeptide with four distinct functional regions: (i) an $\mathrm{N}$-terminal region involved in transcriptional activation (ligand-independent activation function 1 (AF1) domain), (ii) a DNA-binding domain composed of two zinc fingers that

Corresponding Author: Tiffany A. Traina, M.D., Address: Breast Medicine Service, Memorial Sloan Kettering Cancer Center, Weill Cornell Medical College, Evelyn H. Lauder Breast Center, 300 East 66th Street, New York, NY 10065, trainat@ mskcc.org.

Publisher's Disclaimer: This is a PDF file of an unedited manuscript that has been accepted for publication. As a service to our customers we are providing this early version of the manuscript. The manuscript will undergo copyediting, typesetting, and review of the resulting proof before it is published in its final citable form. Please note that during the production process errors may be discovered which could affect the content, and all legal disclaimers that apply to the journal pertain. 
interacts with androgen response elements, (iii) a ligand-binding domain (LBD) to which androgens and antiandrogens bind which includes an androgen-dependent activation function 2 domain at the C-terminus), and (iv) a hinge region that connects the DNA-binding domain and LBD, and controls nuclear localization. Unbound AR resides primarily in the cytoplasm, bound to chaperone proteins such as heat shock proteins that stabilize the receptor in a conformation that promotes ligand binding. In the presence of androgens (testosterone and ddihydrotestosterone (DHT)), the AR undergoes a series of conformational changes, dissociates from the heat shock proteins, and forms a homodimer that translocates to the nucleus. In the nucleus the AR-complex binds to androgen response elements and recruits co-regulatory activators leading to the activation of target gene transcription[5-7].

Published data supports that the androgen-signaling pathway has a role in breast cancer development. Notably, both growth stimulatory and inhibitory effects of androgens have been described in breast cancer cells lines $[8,9]$. Reconciling these seemingly paradoxical effects is complex. However, emerging data suggests that the function of the AR in breast cancer pathogenesis may be in part dependent on the underlying molecular phenotype of the tumor, the relative coexpression of other hormone receptors, and the hormonal environment. Historically androgens, such as fluoxymesterone, testolactone, and calusterone have been used for the treatment of advanced breast cancer resulting in clinical responses ranging from 18-39\% [10-13]. However, the undesirable virilizing side effects of these agents have limited their routine use in the treatment of breast cancer particularly in the advent of newer, less toxic endocrine agents. In contrast several clinical trials have now demonstrated the activity of anti-androgen therapy for the treatment of AR+ TNBC.[14-16]

The mechanism by which androgen signaling influences hormonal sensitivity and breast carcinogenesis remains incompletely characterized and an area of active research. The following sections will focus on the recent progress made in further understanding this subgroup of TNBC characterized by AR expression, the underlying mechanisms by which AR driven signaling may influence the development of this subtype of breast cancer, and the available clinical data regarding the use of anti-androgen therapy for the treatment AR+ TNBC.

\section{Androgen receptor in the pathogenesis of triple negative breast cancer}

Farmer and colleagues described the existence of a subset of ER/PgR (-) tumors that demonstrated increased androgen signaling, a molecular apocrine gene expression profile, and increased co-expression of HER2, differentiating these tumors from the classic basal phenotype that had previously been described.[17-20] Independently, researchers at Memorial Sloan Kettering Cancer Center identified a similar subset of hormone receptor (HR)-negative, AR+ breast cancer. Analyzing the gene expression profiles of 99 archived primary breast tumors, Doane et al showed that this subgroup, named ER(-) class A, was distinguished by AR expression, a hormonally driven transcription program and androgendependent and estrogen-independent growth. Gene expression signatures were then used to develop a predictive model to identify molecularly similar breast cancers in an independent human breast cancer data set and among breast cancer cell lines. In order to evaluate the functional role of the AR in this subset of tumors, in vitro studies were carried out on the 
MDA-MB-453 breast cancer cell line which has a molecular phenotype similar to that of the ER(-) class A subtype. Exposure to estrogen failed to produce measurable cell growth in the MDA-MB-453 cell line and subsequent exposure to antiestrogens did not have any effect, further reducing the likelihood that growth in this tumor subgroup was estrogen-dependent. In comparison, incubation with the synthetic androgen, R-1881, resulted in cell proliferation which was then abrogated by the AR-inhibitor flutamide, suggesting an AR-dependent mechanism of cell growth in this cell line.[21]

Several other groups have subsequently described this unique subset of HR(-) tumors.[2227] Evaluating over 500 samples from patients with TNBC, Lehmann and colleagues described significant heterogeneity within TNBC. One of the 6 subgroups they described, termed the luminal androgen receptor (LAR) subtype was characterized by high expression of the AR mRNA and protein, AR downstream effectors/coactivators, and luminal cytokeratins/markers, and lacking basal cytokeratin expression. The MDA-MB-453 cell line along with three additional cell lines (SUM185PE, CAL-148, and MFM-223), identified through gene expression profiling to have a molecular phenotype similar to that of LAR tumors, were used to further assess the functional role of the AR in this ER/PgR(-) subset. As anticipated LAR cell lines were significantly more sensitive to bicalutamide exposure then other TNBC cell lines $\left(\mathrm{IC}_{50}=227 \mu \mathrm{M}\right.$ vs IC50>600 $\left.\mu \mathrm{M} ; \mathrm{P}=0.007\right)$. Xenograft murine models representative of LAR breast cancer were significantly growth inhibited when treated with bicalutamide relative to treatment with vehicle control or other agents. Moreover transfection of LAR cell lines with AR siRNA allowed investigators to show decrease in colony formation in the cell lines with knockdown AR expression in comparison to controls (MFM-223=55\%, $\mathrm{P}=0.031$; MDA-MB-453=51\%, $\mathrm{P}=0.004$; $\mathrm{SUM} 185 \mathrm{PE}=42.3 \%$; $\mathrm{P}=0.002$ ). This preclinical work further substantiated the hypothesis that tumor growth in this subtype is dependent on androgen signaling and that AR expression may be necessary for cell survival and proliferation. Subsequently others have studied the effects of next generation AR-inhibitors such as enzalutamide in LAR cell lines and mouse models with similar results.[28] Collectively these data set the stage for investigators to evaluate the utility of targeting the AR-signaling pathway in the treatment of this subgroup of TNBC.

\section{Androgen receptor as a therapeutic target in triple negative breast cancer}

The current standard of care in the treatment of TNBC offers limited therapeutic options aside from traditional cytotoxic chemotherapy agents. Treatments that target the androgensignaling pathway have the potential to allow a greater number of patients with advanced or metastatic TNBC the opportunity to be treated with effective, less toxic endocrine agents.

\section{Monotherapy}

Bicalutamide-Building upon the preclinical work of Doane and colleagues, investigators at MSK led a multicenter, single arm, phase II trial through the Translational Breast Cancer Research Consortium (TBCRC) to test the hypothesis that AR-inhibition with bicalutamide (150 mg daily) had antitumor activity in patients with advanced or metastatic AR+ ER/ PgR(-) breast cancer (NCT00468715/TBCRC011). Bicalutamide is an orally available nonsteroidal competitive AR inhibitor that is approved by the US Food and Drug 
Administration (FDA) for the treatment of advanced prostate cancer in combination with a luteinizing hormone-releasing hormone analogue. The primary endpoint was clinical benefit rate (CBR) defined as complete response (CR), partial response (PR), or stable disease (SD) $>6$ months. More than 450 patients were screened for AR status; $\sim 12 \%$ of patients had $>10 \%$ nuclear expression of AR by immunohistochemical testing of either their primary tumor or a metastatic site. Daily treatment with bicalutamide was well-tolerated and resulted in a CBR of $19 \%$ with a median PFS of 12 weeks in patients in this population. A subset of patients demonstrated prolonged stable disease, measured in years and one patient with unresectable, locally advanced disease following neoadjuvant anthracycline- and taxanebased therapy was able to achieve definitive local therapy following treatment with bicalutamide.[14] These data are a signal of activity for androgen blockade in AR+ ER/PgR - breast cancer and serve as a proof of concept.

The development of novel, next-generation compounds which inhibit AR activity for the treatment of metastatic castrate resistant prostate cancer (mCRPC), such as enzalutamide and abiraterone acetate, led to the hypothesis that they may offer superior clinical outcomes for AR-dependent breast cancers.

Enzalutamide-Enzalutamide is a pure androgen receptor signaling inhibitor. In addition it inhibits androgen receptor nuclear translocation, DNA binding, and coactivator mobilization. Unlike other antiandrogen agents, it has no known agonistic properties. The FDA approved enzalutamide for the treatment of patients with mCRPC who have previously received docetaxel based on a randomized, placebo-controlled, multicenter trial which demonstrated an improvement in overall survival in this population as compared to placebo. Additional phase II studies have shown enzalutamide to be superior to bicalutamide in terms of progression-free survival (PFS) in a similar patient population [29, 30]. In a phase I doseescalation study in patients with metastatic breast cancer (MBC), enzalutamide was found to be well tolerated at $160 \mathrm{mg}$ daily with common treatment-related adverse events including nausea, vomiting and fatigue $[31,32]$.

For patients with AR+ metastatic TNBC, a phase II study of single-agent enzalutamide 160 mg daily has completed accrual (NCT01889238). Similar to TBCRC011 the primary endpoint of this study $\mathrm{CBR}$ defined here as $\mathrm{CR}+\mathrm{PR}+\mathrm{SD}$ at 16 weeks in the evaluable patient population. Individuals with AR staining $>10 \%$ and having one post-baseline assessment available were considered evaluable. The trial met its primary endpoint with a CBR at 16 of $35 \%$ and a median PFS of 14.7 weeks. In addition two CRs and five PRs were reported in response to enzalutamide therapy. The most common adverse events were fatigue, nausea and decreased appetite. The only common $(5 \%)$ grade 3 or greater adverse event related to study agent was fatigue. There were no serious adverse events reported in this population.

Recognizing that AR by IHC may not be the ideal predictor of benefit to anti-androgen therapy one of the key exploratory endpoints of the study was the development of a biomarker to predict response to enzalutamide in this population. Forty-seven percent of patients in the intention to treat (ITT) population $(n=118)$ were found to be positive for the biomarker (Dx). Stratifying by Dx status in the ITT population, patients with Dx+ TNBC experienced more favorable outcomes in all response measures including CBR16 (39\% vs 
$11 \%$ ), CBR24 (36\% vs 6\%) and median PFS (16.1 weeks vs 8.1 weeks) when compared to their biomarker negative counterparts. This trial further reinforced the potential utility of anti-androgen therapy for the treatment of a subset of patients with TNBC, providing the first objective RECIST-confirmed responses to an AR-antagonist and advancing the development of an assay to help better select patients with TNBC who may benefit from therapy with enzalutamide.

The ENDEAR study is a phase III trial evaluating the efficacy enzalutamide in patients with $\mathrm{Dx}+\mathrm{TNBC}$. Unlike prior trials in this patient population eligibility is not based on AR expression by IHC but instead on the novel gene expression profile developed as a companion diagnostic during the phase II trial described above. Patients with DX+ TNBC will be randomized in a 1:1:1 to receive enzalutamide monotherapy or paclitaxel+placebo or dual therapy with paclitaxel+enzalutamide. The primary efficacy endpoint is PFS. The trial is expected to begin enrollment in late 2016.

Based on the encouraging data available in the metastatic setting, a phase II study is currently enrolling patients to evaluate the feasibility of enzalutamide therapy in patients with early stage, AR+ TNBC (NCT02750358; Table 1). The use of extended adjuvant oral endocrine therapy is a standard of care for the treatment of patients with ER+ early stage breast cancer based on improvements in disease free and overall survival. However patients with AR+ TNBC do not benefit from anti-estrogen therapy and available cytotoxic therapies remain unfortunately inadequate. Ultimately the aim will be to assess whether patients with AR+ TNBC may similarly benefit from an adjuvant antiandrogen approach as these tumors appear to be driven by AR-signaling rather than ER-signaling. However the primary endpoint for this phase II trial is feasibility defined by the discontinuation rate of enzalutamide for 1 year due to toxicity, withdrawal of consent, or other causes related to tolerability.

Abiraterone Acetate-Abiraterone acetate is a potent, orally available, selective inhibitor of both 17a-hydroxylase and c17, 20-lyase, which targets adrenal and tumor intracrine androgen biosynthesis. Abiraterone acetate in combination with prednisone is FDA approved for the treatment of advanced prostate cancer in men who are both chemotherapynaïve disease and following progression on docetaxel. Based on the mechanism of action of abiraterone acetate as a CYP17 inhibitor, there is a rationale for studying its potential benefit in $\mathrm{AR}+\mathrm{TNBC}$, as reduced levels of androgens are expected from upstream inhibition of the steroid synthesis pathway. To assess the safety and efficacy of abiraterone acetate in combination with prednisone a phase II multicenter trial of was conducted through the French cooperative group UNICANCER in women with metastatic or inoperable locally advanced AR+ TNBC. One hundred thirty-eight patients with adequate tissue were AR tested of which 53 (38.4\%) were AR-positive and triple-negative. From July 2013-December 2014, Bonnefoi and colleagues treated 34 women with AR+ TNBC. Ultimately 30 patients were deemed eligible and evaluable for the primary endpoint of CBR at 6 months. Patients were considered evaluable if they completed at least one cycle of therapy and underwent having at least one disease assessment at eight weeks after initiating study treatment. CBR at 6 months was $20.0 \%$ (95\% CI: 7.7-38.6\%), including 1 CR and 5 SD $\geq 6$ months. Median PFS was 2.8 months (95\% CI: 1.7-5.4\%). Similar to TBCRC011, the subgroup of patients 
who achieved clinical benefit were generally older, without visceral involvement, and had received less lines of therapy in the metastatic setting. As expected, the most common drugrelated adverse events included fatigue, hypertension, hypokalemia and nausea. While grade 3 adrenal insufficiency, hypokalemia, and hypertension were reported in this population, there were no grade 4 or 5 adverse events. Although the study did not meet its primary endpoint, 2 objective responses were documented in this population suggesting that coadministration of abiraterone acetate and prednisone may be beneficial for a select population of patients with molecular apocrine-like tumors.

\section{Ongoing clinical trials targeting the AR-signaling pathway}

The development of agents that target the AR-signaling pathway for the treatment of TNBC is an area active investigation. A number of other novel antiandrogens are currently undergoing evaluation in clinical trials either as monotherapy or in combination with other targeted agents (Table 1).

Seviteronel-Another androgen-directed therapy under investigation for the treatment of $\mathrm{AR}+\mathrm{TNBC}$ is seviteronel. Seviteronel is an oral, CYP17-L inhibitor and AR antagonist with activity in castration resistant prostate cancer (CRPC) at a dose of $750 \mathrm{mg}$ nightly. Preclinical data demonstrates that seviteronel potently inhibits the growth of ER(-)/AR(+) MDA-MB-453 breast cancer in a dose-dependent manner. The phase I dose-escalation study in patients with advanced or metastatic breast cancer has been completed. Preliminary pharmacokinetic data presented at the annual American Society of Clinical Oncology (ASCO) meeting in 2016 suggests seviteronel bioavailability may be gender-specific and related to body mass/surface area in women. Overall seviteronel was well-tolerated and 450 $\mathrm{mg}$ was selected as the recommended phase II dose in women[33]. The phase II in this population is currently enrolling patients (NCT02580448).

\section{Dual inhibition of AR and other signaling pathways}

Investigation of the AR pathway in the development of breast cancer has led to the identification of additional signal transduction pathways with the potential to regulate AR downstream activity. This network of signaling pathways, (e.g. PI3K/AKT/mTOR and cellcycle regulators) are hypothesized to work in concert with AR to regulate cell proliferation and tumor growth. Several clinical trials have been developed to test whether dual-inhibition of these signaling pathways will improve outcomes for patients with androgen-drive TNBC.

Dual CDK4/6 and AR inhibition-The rationale for combining palbociclib with AR blockade stems from published data in patients with advanced ER+ breast cancer. Palbociclib is an orally administered potent and highly selective reversible inhibitor of CDK4/6 which been shown to significantly improve median PFS in two large phase III studies in combination with either letrozole (PALOMA-2)[34] or fulvestrant (PALOMA-3) [35] when compared to monotherapy for the treatment of postmenopausal pts with ER+ MBC. Palbociclib prevents cellular DNA synthesis by prohibiting progression of the cell cycle from G1 into the S phase, as demonstrated in laboratory models [36]. Preclinical data indicate that the compound may be expected to have direct cytotoxic activity as well as potential for growth arrest. Arrest in the G1 phase is known to cause cell death in some 
tumor types, as demonstrated by this and other G1 arresting agents (e.g., tamoxifen). Moreover, CDK inhibitors like palbociclib have the potential to enhance the effects of conventional therapies. For example, palbociclib is synergistic with tamoxifen in ERpositive cell lines with a mean combination index $<1$ across clinically relevant drug concentrations [37]. In addition, preclinical data found the luminal subtype, elevated cyclin D1 (CCND1) and Rb protein expression and reduced p16 expression as associated with sensitivity to palbociclib [37]. AR+ TNBC expresses a luminal profile. Palbociclib has been shown to reduce growth of MDA-MB-453 breast cancer cells via reduced $\mathrm{Rb}$ phosphorylation; it inhibits the incorporation of thymidine into the DNA of RB-positive breast cancer cells [37]. In addition, AR+ TNBC has intact Rb protein, the target of palbociclib activity [37]. Based on these collective data, a phase I/II evaluating the combination of bicalutamide and palboiclib in women with AR+ TNBC is currently enrolling patients (NCT02605486) [38].

Dual PI3K and AR inhibition-The PI3K/AKT/mTOR signaling pathway is integral in cell growth and survival and has been implicated in breast cancer development. Activating mutations in PI3KCA have been described in association with androgen receptor AR positivity in breast tumors $[25,39,40]$. Association between AR and pAKT, a known downstream target of this pathway, has also been reported $(\mathrm{P}=0.003)$ [41]. The interaction between AR and the PI3K signaling pathway has been a particular focus of investigation within the AR+ TNBC population as a potential therapeutic target. Lehmann et al. demonstrated significantly increased sensitivity of LAR cell lines, which are frequently associated with PIK3CA mutations, to NVP-BEZ235 (a dual PI3K/mTOR inhibitor) when compared to the basal-like subtype, $\left(\mathrm{IC}_{50}=37 \mathrm{nM}\right.$ vs $\left.\mathrm{IC}_{50}=116 \mathrm{nM}, \mathrm{P}=0.01\right)$ [25]. These preclinical findings have been translated into a multicenter phase I/II trial of enzalutamide in combination with taselisib for the treatment of patients with advanced AR+ TNBC through the TBCRC (NCT02457910).

\section{Conclusion}

The characterization of "TNBC" as a unique breast cancer subtype is a relatively recent development. In the intervening years, advances in molecular profiling have led to the categorization of TNBC into multiple reproducible subgroups distinguished by distinct gene signatures. Clinically the use of the term TNBC highlights the shortcomings of this classification as this cohort is defined largely by what it lacks rather than by molecular subclassification. Cost and complexity of gene expression profiling limit its everyday use in the clinical setting. However these innovations have led to the identification of molecular targets and continue to inform the development of novel therapeutic agents. Several groups have described a subtype of breast cancer which demonstrates increased AR expression and low expression of ER, PR, and HER2. And now preclinical and clinical data have confirmed the potential for androgen blockade as a treatment option for AR+ TNBC. Ongoing research in this area has the potential to ultimately allow a greater number of patients with breast cancer the option of safe and effective endocrine therapy, delaying the need for cytotoxic chemotherapy. 


\section{References}

1. Brenton JD, Carey LA, Ahmed AA, Caldas C. Molecular classification and molecular forecasting of breast cancer: ready for clinical application? J Clin Oncol. 2005; 23:7350-7360. [PubMed: 16145060]

2. American Cancer Society. Cancer Facts \& Figures 2016. Atlanta: American Cancer Society; 2016.

3. Dent R, Trudeau M, Pritchard KI, et al. Triple-negative breast cancer: clinical features and patterns of recurrence. Clin Cancer Res. 2007; 13:4429-4434. [PubMed: 17671126]

4. Bauer KR, Brown M, Cress RD, et al. Descriptive analysis of estrogen receptor (ER)-negative, progesterone receptor (PR)-negative, and HER2-negative invasive breast cancer, the so-called triplenegative phenotype: a population-based study from the California cancer Registry. Cancer. 2007; 109:1721-1728. [PubMed: 17387718]

5. Claessens F, Denayer S, Van Tilborgh N, et al. Diverse roles of androgen receptor (AR) domains in AR-mediated signaling. Nucl Recept Signal. 2008; 6:e008. [PubMed: 18612376]

6. Gelmann EP. Molecular biology of the androgen receptor. J Clin Oncol. 2002; 20:3001-3015. [PubMed: 12089231]

7. Lee HJ, Chang C. Recent advances in androgen receptor action. Cell Mol Life Sci. 2003; 60:16131622. [PubMed: 14504652]

8. Birrell SN, Hall RE, Tilley WD. Role of the androgen receptor in human breast cancer. J Mammary Gland Biol Neoplasia. 1998; 3:95-103. [PubMed: 10819508]

9. Toth-Fejel S, Cheek J, Calhoun K, et al. Estrogen and androgen receptors as comediators of breast cancer cell proliferation: providing a new therapeutic tool. Arch Surg. 2004; 139:50-54. [PubMed: 14718276]

10. Goldenberg IS, Waters N, Ravdin RS, et al. Androgenic therapy for advanced breast cancer in women. A report of the cooperative breast cancer group. JAMA. 1973; 223:1267-1268. [PubMed: 4739330]

11. Ingle JN, Twito DI, Schaid DJ, et al. Combination hormonal therapy with tamoxifen plus fluoxymesterone versus tamoxifen alone in postmenopausal women with metastatic breast cancer. An updated analysis. Cancer. 1991; 67:886-891. [PubMed: 1991261]

12. Manni A, Arafah BM, Pearson OH. Androgen-induced remissions after antiestrogen and hypophysectomy in stage IV breast cancer. Cancer. 1981; 48:2507-2509. [PubMed: 7296499]

13. Tormey DC, Lippman ME, Edwards BK, Cassidy JG. Evaluation of tamoxifen doses with and without fluoxymesterone in advanced breast cancer. Ann Intern Med. 1983; 98:139-144. [PubMed: 6824247]

14. Gucalp A, Tolaney S, Isakoff SJ, et al. Phase II trial of bicalutamide in patients with androgen receptor-positive, estrogen receptor-negative metastatic Breast Cancer. Clin Cancer Res. 2013; 19:5505-5512. [PubMed: 23965901]

15. Traina TA, Miller K, Yardley DA, et al. Results from a phase 2 study of enzalutamide (ENZA), an androgen receptor (AR) inhibitor, in advanced AR+ triple-negative breast cancer (TNBC). ASCO Meeting Abstracts. 2015; 33:1003.

16. Bonnefoi H, Grellety T, Tredan O, et al. A phase II trial of abiraterone acetate plus prednisone in patients with triple-negative androgen receptor positive locally advanced or metastatic breast cancer (UCBG 12-1). Ann Oncol. 2016; 27:812-818. [PubMed: 27052658]

17. Perou CM, Sorlie T, Eisen MB, et al. Molecular portraits of human breast tumours. Nature. 2000; 406:747-752. [PubMed: 10963602]

18. Sorlie T, Tibshirani R, Parker J, et al. Repeated observation of breast tumor subtypes in independent gene expression data sets. Proc Natl Acad Sci U S A. 2003; 100:8418-8423. [PubMed: 12829800]

19. Sotiriou C, Neo SY, McShane LM, et al. Breast cancer classification and prognosis based on gene expression profiles from a population-based study. Proc Natl Acad Sci U S A. 2003; 100:1039310398. [PubMed: 12917485]

20. Farmer P, Bonnefoi H, Becette V, et al. Identification of molecular apocrine breast tumours by microarray analysis. Oncogene. 2005; 24:4660-4671. [PubMed: 15897907] 
21. Doane AS, Danso M, Lal P, et al. An estrogen receptor-negative breast cancer subset characterized by a hormonally regulated transcriptional program and response to androgen. Oncogene. 2006; 25:3994-4008. [PubMed: 16491124]

22. Sanga S, Broom BM, Cristini V, Edgerton ME. Gene expression meta-analysis supports existence of molecular apocrine breast cancer with a role for androgen receptor and implies interactions with ErbB family. BMC Med Genomics. 2009; 2:59. [PubMed: 19747394]

23. Weigelt B, Horlings HM, Kreike B, et al. Refinement of breast cancer classification by molecular characterization of histological special types. J Pathol. 2008; 216:141-150. [PubMed: 18720457]

24. Teschendorff AE, Miremadi A, Pinder SE, et al. An immune response gene expression module identifies a good prognosis subtype in estrogen receptor negative breast cancer. Genome Biol. 2007; 8:R157. [PubMed: 17683518]

25. Lehmann BD, Bauer JA, Chen X, et al. Identification of human triple-negative breast cancer subtypes and preclinical models for selection of targeted therapies. J Clin Invest. 2011; 121:2750 2767. [PubMed: 21633166]

26. Burstein MD, Tsimelzon A, Poage GM, et al. Comprehensive genomic analysis identifies novel subtypes and targets of triple-negative breast cancer. Clin Cancer Res. 2015; 21:1688-1698. [PubMed: 25208879]

27. Jezequel P, Loussouarn D, Guerin-Charbonnel C, et al. Gene-expression molecular subtyping of triple-negative breast cancer tumours: importance of immune response. Breast Cancer Res. 2015; 17:43. [PubMed: 25887482]

28. Cochrane DR, Bernales S, Jacobsen BM, et al. Role of the androgen receptor in breast cancer and preclinical analysis of enzalutamide. Breast Cancer Res. 2014; 16:R7. [PubMed: 24451109]

29. Shore ND, Chowdhury S, Villers A, et al. Efficacy and safety of enzalutamide versus bicalutamide for patients with metastatic prostate cancer (TERRAIN): a randomised, double-blind, phase 2 study. The Lancet Oncology. 17:153-163.

30. Penson DF, Armstrong AJ, Concepcion R, et al. Enzalutamide Versus Bicalutamide in CastrationResistant Prostate Cancer: The STRIVE Trial. J Clin Oncol. 2016; 34:2098-2106. [PubMed: 26811535]

31. Elias A, Burris H, Patel M, et al. Abstract P1-16-05: MDV3100-08: A phase 1 study evaluating the safety and pharmacokinetics of enzalutamide plus fulvestrant in women with advanced hormone receptor-positive breast cancer. Cancer Research. 2016; 76:P1-16-05-P11-16-05.

32. Traina T, Yardley D, Patel M, et al. Abstract PD3-6: A phase 1 open-label, dose-escalation study evaluating the safety, tolerability, and pharmacokinetics of enzalutamide (previously MDV3100) alone or in combination with an aromatase inhibitor in women with advanced breast cancer. Cancer Research. 2013; 73:PD3-6-PD3-6.

33. Bardia A, Dacosta NA, Gabrail NY, et al. Phase (Ph) 1 study of oral seviteronel (VT-464), a dual CYP17-Lyase (L) inhibitor and androgen receptor (AR) antagonist, in patients (pts) with advanced $\mathrm{AR}+$ triple negative $(\mathrm{TNBC})$ or estrogen receptor $(\mathrm{ER})+$ breast cancer (BC). ASCO Meeting Abstracts. 2016; 34:1088.

34. Finn RS, Martin M, Rugo HS, et al. PALOMA-2: Primary results from a phase III trial of palbociclib $(\mathrm{P})$ with letrozole $(\mathrm{L})$ compared with letrozole alone in postmenopausal women with ER+/HER2- advanced breast cancer (ABC). ASCO Meeting Abstracts. 2016; 34:507.

35. Turner NC, Ro J, André F, et al. Palbociclib in Hormone-Receptor Positive Advanced Breast Cancer. New England Journal of Medicine. 2015; 373:209-219. [PubMed: 26030518]

36. Cadoo KA, Gucalp A, Traina TA. Palbociclib: An evidence based review of its potential in the treatment of breast cancer. Breast Cancer: Targets and Therapy. In Press.

37. Finn RS, Dering J, Conklin D, et al. PD 0332991, a selective cyclin D kinase 4/6 inhibitor, preferentially inhibits proliferation of luminal estrogen receptor-positive human breast cancer cell lines in vitro. Breast Cancer Res. 2009; 11:R77. [PubMed: 19874578]

38. Gucalp A, Proverbs-Singh TA, Corben A, et al. Phase I/II trial of palbociclib in combination with bicalutamide for the treatment of androgen receptor (AR)+ metastatic breast cancer (MBC). ASCO Meeting Abstracts. 2016; 34:TPS1103. 
39. Doane ASMM, Zhang L, Hudis C, Gerald WL. PIK3CA mutation is frequent in class A estrogen receptor negative breast cancer and contributes to the distinct molecular profile. Breast Cancer Research and Treatment. 2006:S293.

40. Gonzalez-Angulo AM, Stemke-Hale K, Palla SL, et al. Androgen receptor levels and association with PIK3CA mutations and prognosis in breast cancer. Clin Cancer Res. 2009; 15:2472-2478. [PubMed: 19276248]

41. Aleskandarany MA, Rakha EA, Ahmed MA, et al. Clinicopathologic and molecular significance of phospho-Akt expression in early invasive breast cancer. Breast Cancer Res Treat. 2011; 127:407416. [PubMed: 20617378] 


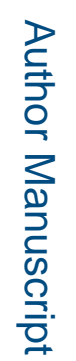

\begin{tabular}{|c|c|c|c|c|c|c|c|c|c|}
\hline 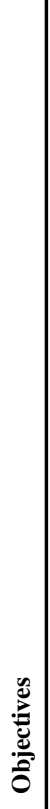 & 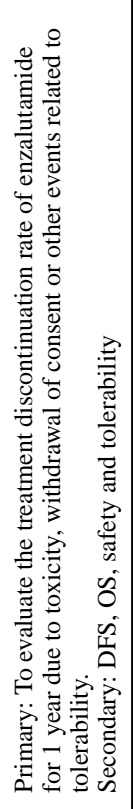 & 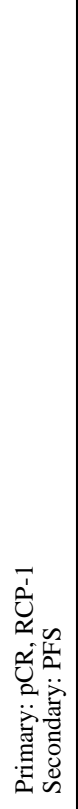 & 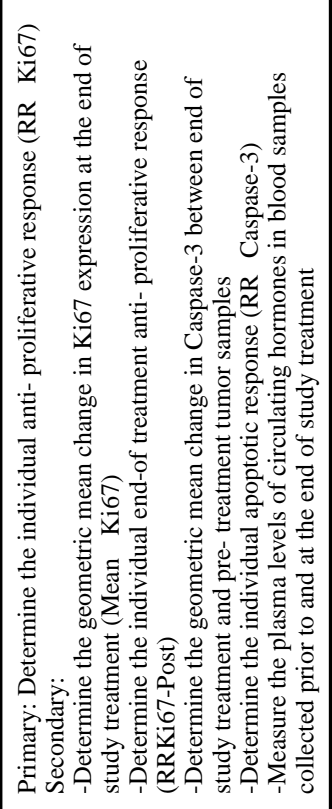 & 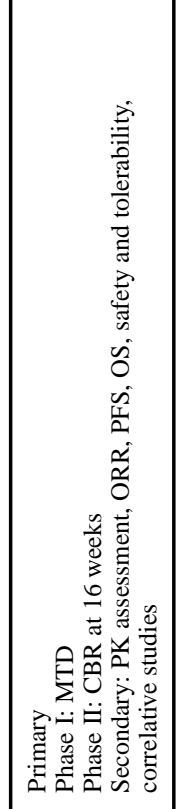 & 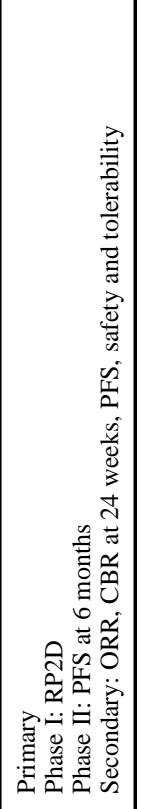 & 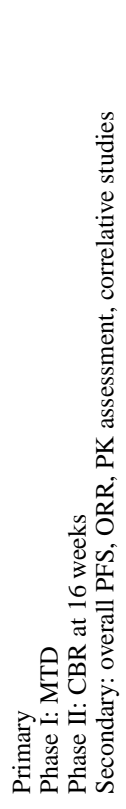 & 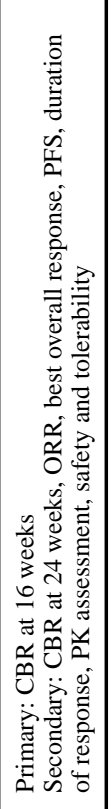 & 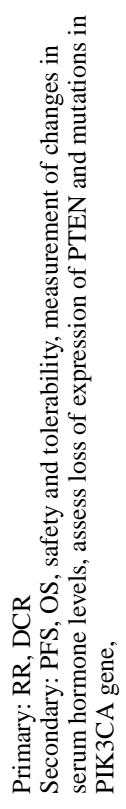 & 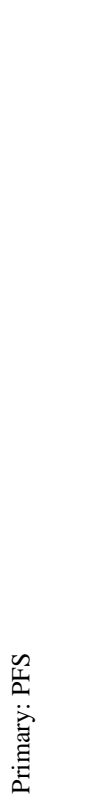 \\
\hline 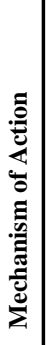 & 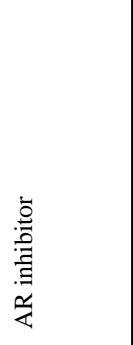 & 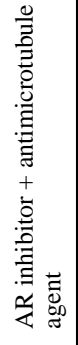 & 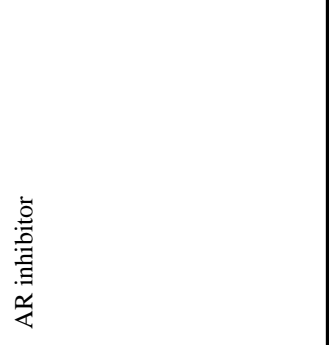 & 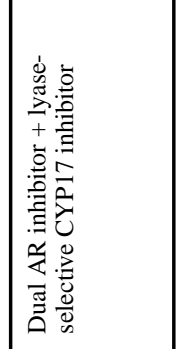 & 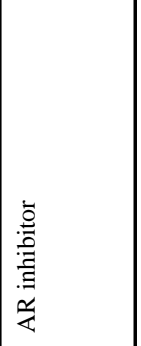 & 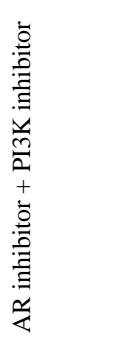 & 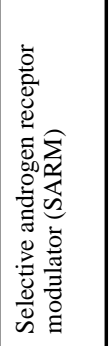 & 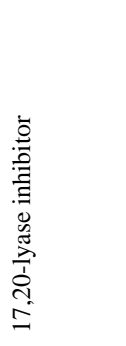 & 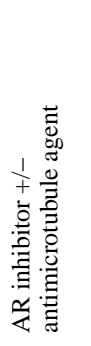 \\
\hline 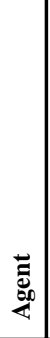 & 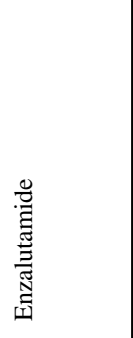 & 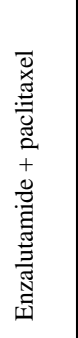 & 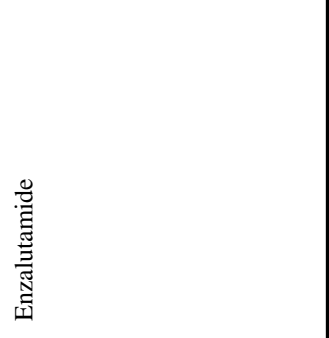 & 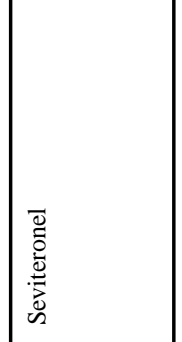 & 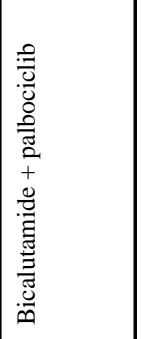 & 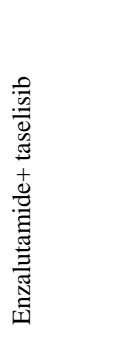 & 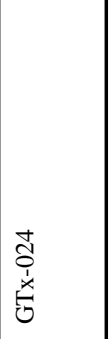 & $\begin{array}{l}\bar{\Xi} \\
\overline{0} \\
\bar{\Xi} \\
0\end{array}$ & 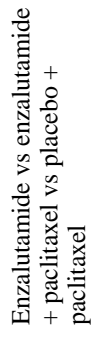 \\
\hline 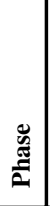 & 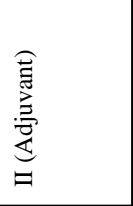 & 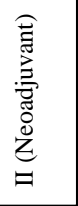 & 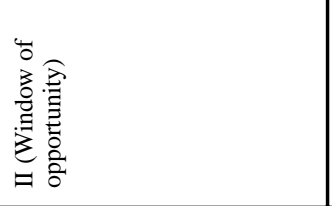 & $\Xi$ & $\Xi$ & $\Xi$ & $=$ & $=$ & $\Xi$ \\
\hline 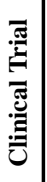 & 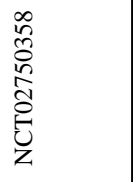 & 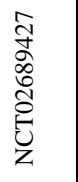 & $\begin{array}{l}0 \\
\infty \\
0 \\
0 \\
0 \\
0 \\
0 \\
0 \\
z \\
z\end{array}$ & \begin{tabular}{l} 
ơ \\
\multirow{0}{*}{} \\
$\infty$ \\
0 \\
0 \\
0 \\
$z$ \\
$z$
\end{tabular} & $\begin{array}{l}0 \\
\infty \\
0 \\
0 \\
0 \\
0 \\
0 \\
0 \\
0 \\
z \\
z\end{array}$ & 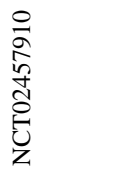 & 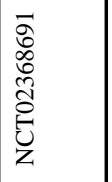 & 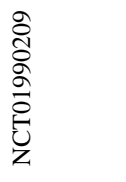 & 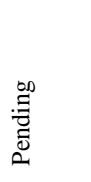 \\
\hline 垔 & 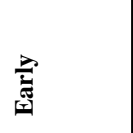 & & & & & & & & \\
\hline
\end{tabular}

Curr Probl Cancer. Author manuscript; available in PMC 2017 September 20. 


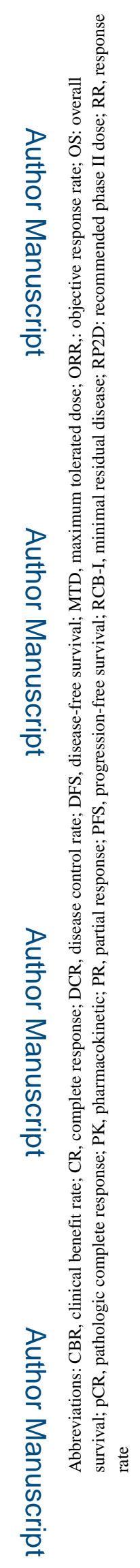

